Research Paper

\title{
Evidence of association between single-nucleotide polymorphisms in lipid metabolism-related genes and type 2 diabetes mellitus in a Chinese population
}

\author{
Yiping $\mathrm{Li}^{1 *}$, Siqi $\mathrm{He}^{1,2^{*}}$, Chuanyin $\mathrm{Li}^{3}$, Keyu Shen ${ }^{3}$, Man Yang1, Wenyu Tao ${ }^{1}$, Ying Yang ${ }^{1}$, Li Shi ${ }^{\circledR}{ }^{\bowtie}$ and \\ Yufeng $\mathrm{Yao}^{3 凶}$ \\ 1. Department of Endocrinology and Metabolism, The Second People's Hospital of Yunnan Province \& The Affiliated Hospital of Yunnan University, \\ Kunming 650021, Yunnan, China. \\ 2. Dali University, Dali 671000, Yunnan, China. \\ 3. Institute of Medical Biology, Chinese Academy of Medical Sciences \& Peking Union Medical College, Kunming 650118, Yunnan, China. \\ 4. Department of Medicine, Dentistry and Healthy Science, The University of Melbourne, Melbourne VIC3010, Australia. \\ *These authors contributed equally to this work.
}

$\square$ Corresponding authors: Prof. Li Shi, Institute of Medical Biology, Chinese Academy of Medical Sciences \& Peking Union Medical College, Kunming 650118, Yunnan, China. E-mail address: shili.imb@gmail.com; Dr. Yufeng Yao, Institute of Medical Biology, Chinese Academy of Medical Sciences \& Peking Union Medical College, Kunming 650118, Yunnan, China. E-mail address: leoyyf@gmail.com or yufeng_yao@imbcams.com.cn.

(C) The author(s). This is an open access article distributed under the terms of the Creative Commons Attribution License (https://creativecommons.org/licenses/by/4.0/). See http://ivyspring.com/terms for full terms and conditions.

Received: 2020.09.08; Accepted: 2020.11.09; Published: 2021.01.01

\begin{abstract}
Background: Type 2 diabetes mellitus (T2DM) is a complex chronic metabolic disorder triggered by insulin resistance in peripheral tissues. Evidence has shown that lipid metabolism and related genetic factors lead to insulin resistance. Hence, it is meaningful to investigate the association between single-nucleotide polymorphisms (SNPs) in lipid metabolism-related genes and T2DM.

Methods: A total of 1,194 subjects with T2DM and 1,274 Non-diabetic subjects (NDM) were enrolled. Five SNPs in three genes (rs864745 in JAZFI, rs35767 in IGFI, and rs4376068, rs4402960, and rs6769511 in IGF2BP2) that contribute to insulin resistance involving lipid metabolism were genotyped using the MassArray method in a Chinese population.

Results: The allele and genotypes of $\mathrm{r} 56769511$ in IGF2BP2 were associated with T2DM ( $\mathrm{P}=0.009$ and $\mathrm{P}=0.002$, respectively). In inheritance model analysis, compared with the $\mathrm{T} / \mathrm{T}-\mathrm{C} / \mathrm{T}$ genotype, the $\mathrm{C} / \mathrm{C}$ genotype of rs6769511 in IGF2BP2 was a risk factor for the development of T2DM ( $<<0.001$, odds ratio [OR] $=1.76$; $95 \%$ confidence interval [Cl]: 1.29-2.42). Haplotype analysis revealed associations of the rs4376068rs4402960-rs6769511 haplotypes in IGF2BP2 with the development of T2DM ( $P=0.015)$. Additionally, rs4376068C-rs4402960T-rs6769511C was a risk haplotype for T2DM (OR=1.179; 95\% Cl: 1.033-1.346).

Conclusion: The rs6769511 in IGF2BP2 was associated with T2DM susceptibility, and the rs4376068rs4402960-rs6769511 haplotypes in IGF2BP2 was associated with the development of T2DM in a Chinese population.
\end{abstract}

Key words: Polymorphisms; Chinese population; lipid metabolism-related genes; T2DM

\section{Introduction}

Type 2 diabetes mellitus (T2DM) is a chronic multifactorial hyperglycaemic disease characterised by insulin resistance and impaired insulin secretion. Compared with insulin secretion, insulin resistance is the basis and major feature of T2DM [1] and is an inherited defect that initiates the diabetic condition in the majority of patients with T2DM [2]. Accumulated data have revealed that lipid abnormalities are associated with insulin resistance and contribute to T2DM [3, 4]. Studies have also revealed lipid metabolism-related genes and their single-nucleotide polymorphisms (SNPs) associated with insulin resistance and the development of T2DM [5-12].

Recent evidence has shown that juxtaposed with another zinc finger gene 1 (JAZF1), insulin-like growth factor 1 (IGF1), and insulin-like growth factor 
2 mRNA-binding protein 2 (IGF2BP2), which are expressed in insulin-resistant tissue, are involved in lipid metabolism and are associated with insulin resistance $[7,8,13-18]$. In adipocytes, JAZF1 inhibits the accumulation of fatty acids and triglycerides [15]. In the liver, JAZF1 enhances fatty acid $\beta$-oxidation [16]. IGF-1 downregulates free fatty acid and increases fatty acid $\beta$ oxidation during fasting [9]. Hepatic IGF2BP2 deficiency leads to a progressive deposition of hepatic triglycerides and elevated circulating triglycerides due to reduction of fatty acid oxidation, while global IGF2BP2 deficiency is accompanied by reduced circulating fatty acids and triglycerides [13, 14].

In addition to the indirect role of JAZF1, IGF1, and IGF2BP2 in insulin resistance involving lipid metabolism, the three proteins can also have direct effects on glucose metabolism [5, 16, 19]. The genes encoding these proteins, as well as their SNPs, have been associated with T2DM and metabolic traits [6, 11, 20-26]. For example, Long et al. reported that rs6769511C in IGF2BP2 was associated with the risk of T2DM in African Americans [22]. Then, Kuo et al. also observed that the rs6769511C allele was associated with T2DM in a Chinese Han population [26].

In the current study, 1,194 patients with T2DM and 1,274 Non-diabetic (NDM) subjects in a Chinese population were enrolled. Five SNPs in three genes (rs864745 in JAZF1, rs35767 in IGF1, and rs4376068, rs4402960, and rs6769511 in IGF2BP2) contributing to insulin resistance involving lipid metabolism were genotyped. We also investigated the association of the five SNPs and metabolic traits in the T2DM and NDM groups in the Chinese population.

\section{Materials and Methods}

\section{Ethics statement}

This study was approved by the Institutional Review Board of the Second People's Hospital of Yunnan Province prior to beginning the study. The study protocols complied with the principles expressed in the Helsinki Declaration of 1975, which was revised in 2008. Written informed consent was obtained from each participant.

\section{Subjects}

A total of 1,194 patients (762 men and 432 women) diagnosed with T2DM at the Second People's Hospital of Yunnan Province from February 2017 to July 2019 were enrolled. The diagnostic standard of T2DM conformed to the World Health Organization criteria published in 1999 and the American Diabetes Association guidelines of 2020 [27]. Insulin secretion and C-peptide levels were measured to exclude subjects with type 1 diabetes. For subjects $<40$ years old, islet cell autoantibodies and glutamic acid decarboxylase autoantibodies were detected to exclude autoantibody positive subjects. Gestational women were not included. Specific types of diabetes (e.g., glucocorticoid-induced diabetes) were excluded based on medical history. The NDM group included 1,274 subjects (782 men and 492 women) with no family history of diabetes mellitus. These subjects were recruited from among individuals undergoing routine health check-ups at the Second People's Hospital of Yunnan Province. Subjects with impaired glucose tolerance and/or glycosylated haemoglobin (HbA1C) levels $\geq 5.7 \%$ were excluded from the NDM group [27]. Subjects with hypertension or coronary heart disease were also excluded. All participants described themselves as being of Han ethnicity.

\section{Laboratory measurements}

Venous blood samples were collected the morning after the subjects had fasted for 12 hours. Fasting plasma glucose (FPG), total cholesterol (TC), triglycerides (TG), high-density lipoprotein cholesterol (HDL-C), low-density lipoprotein cholesterol (LDL-C), and HbA1C were measured. All laboratory measurements were performed using a 7600-020 automatic analyser (Hitachi, Tokyo, Japan).

\section{Selection and genotyping of SNPs}

In the current study, we focused on three genes (JAZF1, IGF1, and IGF2BP2) involved in lipid metabolism, which have been shown to affect insulin resistance [5-11, 13-18]. The SNP rs864745 in JAZF1 is located in intron $1[28,29]$ and rs35767 is located in the IGF1 promoter [30]. The three SNPs (rs4376068, rs4402960, and rs6769511) of IGF2BP2 are all located in intron $2[6,31]$.

The QIAamp Blood Mini Kit (Qiagen, Hilden, Germany) was used to extract genomic DNA from peripheral lymphocytes. The aforementioned five SNPs in the three genes were genotyped using the MassArray Analyser 4.0 (Agena Bioscience, Inc., San Diego, CA, USA) as previously described [32]. Matrixassisted laser desorption/ionization time-of-flight mass spectrometry (MALDI-TOF MS) was performed using a device from Agena Bioscience. SNP genotyping was performed using a SpectroCHIP array, and the raw genotyping data were obtained using TYPER4.0 software (Agena Bioscience).

\section{Statistical analyses}

Age, glucose, and lipid metabolic parameters (TC, HDL-C, TG, LDL-C, FPG, and HbA1C) were compared between the T2DM and NDM groups using Student's $t$ test. Sex in the T2DM and NDM groups was compared using the Chi-square test. All polymorphic loci were tested for deviation from the 
Hardy-Weinberg equilibrium in the NDM group with a threshold of 0.05 . Risks were estimated by odds ratios (OR) with $95 \%$ confidence intervals $(95 \% \mathrm{CI})$. Linkage disequilibrium (LD) among these SNPs was estimated. The LD coefficient D was calculated using SHEsis software [33, 34]. The distribution and differences in the haplotypes between the T2DM and NDM groups were determined using SHEsis software. Haplotype frequencies $<0.03$ were ignored in the analysis $[33,34]$. The association between each SNP and T2DM was analysed for mode of inheritance using SNPStats [35]. Power-analysis was performed using power and sample size calculations [36]. Bonferroni correction for multiple comparisons was used for each SNP and haplotype.

\section{Results}

\section{Subject characteristics}

Table 1 lists the clinical characteristics and glucose and lipid metabolic parameters of the enrolled subjects. There were no differences in age or sex between the T2DM and NDM groups, or in age between the male and female subjects in both groups (all $\mathrm{P}>0.05$ ). However, significant differences were evident in the glucose and lipid metabolic parameters (TC, TG, HDL-C, LDL-C, FPG, and HbA1C) between the T2DM and NDM groups (all $\mathrm{P}<0.001$; Table 1 ). T2DM subjects displayed dyslipidaemia and dysglycemia with higher TC, TG, LDL-C, FPG, and HbA1C and lower HDL-C, compared with NDM subjects.

Table 1. Clinical characteristics and glucose and lipid metabolic parameters of the subjects enrolled in the present study

\begin{tabular}{llll}
\hline & $\begin{array}{l}\text { Type 2 diabetes } \\
(\mathrm{T} 2 \mathrm{DM})\end{array}$ & $\begin{array}{l}\text { Non-diabetic } \\
\text { subject (NDM) }\end{array}$ & P value \\
\hline $\mathrm{N}$ & 1,194 & 1,274 & \\
Age, y & $52.490 \pm 12.101$ & $52.764 \pm 10.543$ & 0.550 \\
$\begin{array}{l}\text { Sex (M/F) } \\
\text { Age/M, y }\end{array}$ & $762 / 432$ & $782 / 492$ & 0.211 \\
Age/F, y & $51.366 \pm 12.399$ & $51.715 \pm 10.557$ & 0.552 \\
$\begin{array}{l}\text { Total cholesterol, mmol/L } \\
\text { Triglycerides, mmol/L }\end{array}$ & $54.816 \pm 1.076$ & $4.516 \pm 1.053$ & $<0.001$ \\
$\begin{array}{l}\text { High-density lipoprotein- } \\
\text { cholesterol, mmol/L }\end{array}$ & $1.093 \pm 0.281$ & $1.749 \pm 1.277$ & $<0.001$ \\
$\begin{array}{l}\text { Low-density lipoprotein- } \\
\text { cholesterol, mmol/L }\end{array}$ & $2.794 \pm 0.969$ & $2.632 \pm 0.869$ & $<0.001$ \\
$\begin{array}{l}\text { Fasting plasma glucose, } \\
\text { mmol/L }\end{array}$ & $7.895 \pm 2.503$ & $5.006 \pm 0.527$ & $<0.001$ \\
\begin{tabular}{l} 
HbA1C (\%) \\
\hline
\end{tabular} & $8.990 \pm 2.817$ & $5.138 \pm 0.356$ & $<0.001$ \\
\hline
\end{tabular}

\section{Association of the five SNPs with T2DM}

The allele and genotype frequencies for the five SNPs in the candidate T2DM susceptible genes are listed in Table 2. The genotype frequencies for the five
SNPs in the NDM group were in Hardy-Weinberg equilibrium ( $\mathrm{P}>0.05$; Table 2$)$. The genotype frequency distribution of rs4376068 and rs4402960 in IGF2BP2 showed a different trend between the NDM and T2DM groups $(\mathrm{P}=0.051$ and $\mathrm{P}=0.020$, respectively). The allele frequency distribution of rs4376068 and rs4402960 in IGF2BP2 showed a different trend between the NDM and T2DM groups $(\mathrm{P}=0.022$ and $\mathrm{P}=0.011$, respectively). However, after Bonferroni correction, the alleles and genotypes of these two SNPs showed no significant difference between the NDM and T2DM groups. For the rs6769511 SNP in IGF2BP2, alleles and genotypes showed significant differences between the T2DM and NDM groups after Bonferroni correction $(\mathrm{P}=0.009$ and $\mathrm{P}=0.002$, respectively). The $\mathrm{C}$ allele of rs6769511 was a risk factor for the development of T2DM (OR=1.185; 95\% CI: 1.043-1.347). The allele and genotype frequency distribution of the rs864745 in JAZF1 and rs35767 in IGF1 failed to show an association with T2DM $(\mathrm{P}>0.05)$.

\section{Model of inheritance analysis}

Data on the inheritance models (codominant, dominant, recessive, overdominant, and log-additive) of these SNPs are listed in Tables 3 to 7. The lowest Akaike information criterion (AIC) and Bayesian information criterion (BIC) were used to determine the best fit model for each SNP [35]. For rs6769511 in IGF2BP2, the best-fit inheritance model with the lowest AIC and BIC was recessive. Compared with the $\mathrm{T} / \mathrm{T}-\mathrm{C} / \mathrm{T}$ genotype, the $\mathrm{C} / \mathrm{C}$ genotype was a risk factor for T2DM ( $\mathrm{P}<0.001$, OR=1.76; 95\% CI: 1.29-2.42). In addition, the best-fit inheritance model with the lowest AIC and BIC for rs4376068 and rs4402960 in IGF2BP2 was log-additive. However, both rs4376068 and rs4402960 failed to show significant differences between the T2DM and NDM groups after Bonferroni correction ( $\mathrm{P}=0.020$ and $\mathrm{P}=0.010$, respectively). The other SNPs did not show any association with T2DM $(\mathrm{P}>0.05)$.

\section{LD among the three SNPs in IGF2BP2}

LD is displayed as a pairwise $\mathrm{D}^{\prime}$. The $\mathrm{D}^{\prime}$ values were defined in the range $(-1,1)$, with a value of 1 representing perfect disequilibrium. A $\mathrm{D}^{\prime}$ value $>0.8$ indicated the existence of different loci in the LD. Based on the location of the rs4376068, rs4402960, and rs6769511 SNPs in chromosome 3, the LD among these SNPs was estimated. The three SNPs were in LD $\left(D^{\prime}>0.9\right)$. $\mathrm{D}^{\prime}$ values obtained from all individuals were 0.969 between rs4376068 and rs4402960, 0.936 between rs4376068 and rs6769511, and 0.930 between rs4402960 and rs6769511. 
Table 2. Comparison of genotypic and allelic distribution of five SNPs (rs864745, rs35767, rs4376068, rs4402960 and rs6769511) between the Type 2 diabetes (T2DM) and Non-diabetic subject (NDM)

\begin{tabular}{|c|c|c|c|c|c|c|c|c|c|c|c|}
\hline SNPs & Allele & & $X^{2}$ & $P$ value & Odds ratio $(95 \% \mathrm{CI})$ & Genotypes & & & $\mathrm{X}^{2}$ & $P$ value & $\mathrm{H}-\mathrm{W}$ \\
\hline rs864745 & $\mathrm{T}$ (freq) & $C$ (freq) & & & & $\mathrm{T} / \mathrm{T}$ (freq) & $\mathrm{T} / \mathrm{C}$ (freq) & $\mathrm{C} / \mathrm{C}$ (freq) & & & \\
\hline T2DM & $1869(0.783)$ & $519(0.217)$ & 1.353 & 0.245 & $1.083(0.947-1.238)$ & $736(0.616)$ & $397(0.332)$ & $61(0.051)$ & 3.796 & 0.150 & 0.434 \\
\hline NDM & 1959 (0.769) & $589(0.231)$ & & & & $744(0.584)$ & $471(0.370)$ & $59(0.046)$ & & & 0.152 \\
\hline rs35767 & $\mathrm{G}$ (freq) & A (freq) & & & & $\mathrm{G} / \mathrm{G}$ (freq) & G/A (freq) & A/A (freq) & & & \\
\hline T2DM & $1538(0.644)$ & $850(0.356)$ & 1.590 & 0.207 & 1.077 (0.959-1.210) & $513(0.430)$ & $512(0.429)$ & $169(0.142)$ & 4.278 & 0.118 & 0.025 \\
\hline NDM & $1597(0.627)$ & $951(0.373)$ & & & & $500(0.392)$ & $597(0.469)$ & $177(0.139)$ & & & 0.955 \\
\hline rs4376068 & $\mathrm{C}($ freq $)$ & A (freq) & & & & A/A (freq) & $\mathrm{C} / \mathrm{A}$ (freq) & $C / C$ (freq) & & & \\
\hline T2DM & $627(0.263)$ & $1761(0.737)$ & 5.280 & 0.022 & $1.164(1.022-1.324)$ & $649(0.544)$ & $463(0.388)$ & $82(0.069)$ & 5.933 & 0.051 & 0.963 \\
\hline NDM & $597(0.234)$ & $1951(0.766)$ & & & & $740(0.581)$ & $471(0.370)$ & $63(0.049)$ & & & 0.279 \\
\hline rs4402960 & $\mathrm{T}$ (freq) & $\mathrm{G}$ (freq) & & & & $\mathrm{G} / \mathrm{G}$ (freq) & $\mathrm{T} / \mathrm{G}$ (freq) & $\mathrm{T} / \mathrm{T}$ (freq) & & & \\
\hline T2DM & $645(0.270)$ & $1743(0.730)$ & 6.450 & 0.011 & $1.181(1.039-1.342)$ & $635(0.532)$ & $473(0.396)$ & $86(0.072)$ & 7.779 & 0.020 & 0.871 \\
\hline NDM & $608(0.239)$ & $1940(0.761)$ & & & & $728(0.571)$ & $484(0.380)$ & $62(0.049)$ & & & 0.104 \\
\hline rs6769511 & $\mathrm{C}$ (freq) & $\mathrm{T}$ (freq) & & & & $\mathrm{C} / \mathrm{C}$ (freq) & $\mathrm{C} / \mathrm{T}$ (freq) & $\mathrm{T} / \mathrm{T}$ (freq) & & & \\
\hline T2DM & $655(0.274)$ & $1733(0.726)$ & 6.823 & 0.009 & 1.185 (1.043-1.347) & $107(0.090)$ & $441(0.369)$ & $646(0.541)$ & 12.989 & 0.002 & 0.013 \\
\hline NDM & $616(0.242)$ & $1932(0.758)$ & & & & $67(0.053)$ & $482(0.378)$ & $725(0.569)$ & & & 0.254 \\
\hline
\end{tabular}

Note: Significant threshold after Bonferroni correction for multiple comparisons is less than 0.010 (0.05/5); H-W: Hardy-Weinberg equilibrium; CI: confidence interval.

Table 3. Different inheritance models analysis of the SNP rs6769511 between the Type 2 diabetes (T2DM) and Non-diabetic subject (NDM)

\begin{tabular}{|c|c|c|c|c|c|c|c|}
\hline Model & Genotype & NDM & T2DM & Odds ratio $(95 \% \mathrm{CI})$ & $P$ value & AIC & $\mathrm{BIC}$ \\
\hline \multirow[t]{3}{*}{ Codominant } & $\mathrm{T} / \mathrm{T}$ & $725(56.9 \%)$ & $646(54.1 \%)$ & 1.00 & 0.002 & 3414.2 & 3443.3 \\
\hline & $\mathrm{C} / \mathrm{T}$ & $482(37.8 \%)$ & $441(36.9 \%)$ & $1.03(0.87-1.22)$ & & & \\
\hline & $\mathrm{C} / \mathrm{C}$ & $67(5.3 \%)$ & $107(9 \%)$ & $1.78(1.29-2.47)$ & & & \\
\hline \multirow[t]{2}{*}{ Dominant } & $\mathrm{T} / \mathrm{T}$ & $725(56.9 \%)$ & $646(54.1 \%)$ & 1.00 & 0.160 & 3423 & 3446.3 \\
\hline & $\mathrm{C} / \mathrm{T}-\mathrm{C} / \mathrm{C}$ & $549(43.1 \%)$ & $548(45.9 \%)$ & $1.12(0.96-1.31)$ & & & \\
\hline \multirow[t]{2}{*}{ Recessive } & $\mathrm{T} / \mathrm{T}-\mathrm{C} / \mathrm{T}$ & $1207(94.7 \%)$ & $1087(91 \%)$ & 1.00 & $<0.001$ & 3412.3 & 3435.6 \\
\hline & $\mathrm{C} / \mathrm{C}$ & $67(5.3 \%)$ & $107(9 \%)$ & $1.76(1.29-2.42)$ & & & \\
\hline \multirow[t]{2}{*}{ Overdominant } & $\mathrm{T} / \mathrm{T}-\mathrm{C} / \mathrm{C}$ & $792(62.2 \%)$ & $753(63.1 \%)$ & 1.00 & 0.670 & 3424.8 & 3448.1 \\
\hline & $\mathrm{C} / \mathrm{T}$ & $482(37.8 \%)$ & $441(36.9 \%)$ & $0.96(0.82-1.14)$ & & & \\
\hline Log-additive & --- & -- & --- & $1.18(1.04-1.34)$ & 0.010 & 3418.4 & 3441.6 \\
\hline
\end{tabular}

criterion; CI: confidence interval.

Table 4. Different inheritance models analysis of the SNP rs4402960 between the Type 2 diabetes (T2DM) and Non-diabetic subject (NDM)

\begin{tabular}{|c|c|c|c|c|c|c|c|}
\hline Model & Genotype & NDM & T2DM & Odds ratio $(95 \% \mathrm{CI})$ & $P$ value & AIC & $\mathrm{BIC}$ \\
\hline \multirow[t]{3}{*}{ Codominant } & $\mathrm{G} / \mathrm{G}$ & $728(57.1 \%)$ & $635(53.2 \%)$ & 1.00 & 0.021 & 3419.3 & 3448.4 \\
\hline & $\mathrm{G} / \mathrm{T}$ & $484(38 \%)$ & $473(39.6 \%)$ & $1.12(0.95-1.33)$ & & & \\
\hline & $\mathrm{T} / \mathrm{T}$ & $62(4.9 \%)$ & $86(7.2 \%)$ & $1.58(1.12-2.23)$ & & & \\
\hline \multirow[t]{2}{*}{ Dominant } & $\mathrm{G} / \mathrm{G}$ & $728(57.1 \%)$ & $635(53.2 \%)$ & 1.00 & 0.046 & 3421.0 & 3444.3 \\
\hline & $\mathrm{G} / \mathrm{T}-\mathrm{T} / \mathrm{T}$ & $546(42.9 \%)$ & $559(46.8 \%)$ & $1.18(1.00-1.38)$ & & & \\
\hline \multirow[t]{2}{*}{ Recessive } & G/G-G/T & $1212(95.1 \%)$ & $1108(92.8 \%)$ & 1.00 & 0.016 & 3419.2 & 3442.4 \\
\hline & $\mathrm{T} / \mathrm{T}$ & $62(4.9 \%)$ & $86(7.2 \%)$ & $1.51(1.08-2.11)$ & & & \\
\hline \multirow[t]{2}{*}{ Overdominant } & G/G-T/T & $790(62 \%)$ & $721(60.4 \%)$ & 1.00 & 0.390 & 3424.3 & 3447.5 \\
\hline & $\mathrm{G} / \mathrm{T}$ & $484(38 \%)$ & $473(39.6 \%)$ & $1.07(0.91-1.26)$ & & & \\
\hline Log-additive & --- & 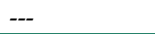 & --- & $1.19(1.04-1.35)$ & 0.010 & 3418.4 & 3441.6 \\
\hline
\end{tabular}

Note: Significant threshold after Bonferroni correction for multiple comparisons is less than 0.010 (0.05/5); AIC: Akaike information criterion; BIC: Bayesian information criterion; CI: confidence interval.

\section{Association of haplotypes of SNPs with T2DM}

According to the $\mathrm{D}^{\prime}$ value $\left(\mathrm{D}^{\prime}>0.8\right)$, the haplotypes of the three SNPs (rs4376068-rs4402960rs6769511) in IGF2BP2 were constructed. The frequency of haplotype rs4376068C-rs4402960Trs6769511C was 0.248 in T2DM and 0.221 in NDM patients. The frequency of haplotype rs4376068Ars4402960G-rs6769511T was 0.708 in T2DM and 0.743 in NDM patients. The haplotypes showed significant differences between the T2DM and NDM groups after Bonferroni correction ( $\mathrm{P}=0.015$; Table 8$)$. rs4376068Crs4402960T-rs6769511C was implicated as a high-risk haplotype of T2DM (OR=1.179, 95\%CI: 1.033-1.346), while rs4376068A-rs4402960G-rs6769511T could be a protective haplotype against T2DM $(\mathrm{OR}=0.848,95 \%$ CI: 0.743-0.969). 
Table 5. Different inheritance models analysis of the SNP rs4376068 between the Type 2 diabetes (T2DM) and Non-diabetic subject (NDM)

\begin{tabular}{|c|c|c|c|c|c|c|c|}
\hline Model & Genotype & NDM & T2DM & Odds ratio $(95 \% \mathrm{CI})$ & $P$ value & AIC & $\mathrm{BIC}$ \\
\hline \multirow[t]{3}{*}{ Codominant } & $\mathrm{A} / \mathrm{A}$ & $740(58.1 \%)$ & $649(54.4 \%)$ & 1.00 & 0.052 & 3421.1 & 3450.2 \\
\hline & $\mathrm{A} / \mathrm{C}$ & $471(37 \%)$ & $463(38.8 \%)$ & $1.12(0.95-1.33)$ & & & \\
\hline & $\mathrm{C} / \mathrm{C}$ & $63(5 \%)$ & $82(6.9 \%)$ & $1.48(1.05-2.09)$ & & & \\
\hline \multirow[t]{2}{*}{ Dominant } & $\mathrm{A} / \mathrm{A}$ & $740(58.1 \%)$ & $649(54.4 \%)$ & 1.00 & 0.059 & 3421.5 & 3444.7 \\
\hline & $\mathrm{A} / \mathrm{C}-\mathrm{C} / \mathrm{C}$ & $534(41.9 \%)$ & $545(45.6 \%)$ & $1.17(0.99-1.37)$ & & & \\
\hline \multirow[t]{2}{*}{ Recessive } & $\mathrm{A} / \mathrm{A}-\mathrm{A} / \mathrm{C}$ & $1211(95 \%)$ & $1112(93.1 \%)$ & 1.00 & 0.045 & 3421.0 & 3444.2 \\
\hline & $\mathrm{C} / \mathrm{C}$ & $63(5 \%)$ & $82(6.9 \%)$ & $1.41(1.01-1.98)$ & & & \\
\hline \multirow[t]{2}{*}{ Overdominant } & $\mathrm{A} / \mathrm{A}-\mathrm{C} / \mathrm{C}$ & $803(63 \%)$ & $731(61.2 \%)$ & 1.00 & 0.340 & 3424.1 & 3447.3 \\
\hline & $\mathrm{A} / \mathrm{C}$ & $471(37 \%)$ & $463(38.8 \%)$ & $1.08(0.92-1.27)$ & & & \\
\hline Log-additive & --- & -- & -- & $1.17(1.02-1.33)$ & 0.020 & 3419.6 & 3442.9 \\
\hline
\end{tabular}

Note: Significant threshold after Bonferroni correction for multiple comparisons is less than 0.010 (0.05/5); AIC: Akaike information criterion; BIC: Bayesian information criterion; CI: confidence interval.

Table 6. Different inheritance models analysis of the SNPrs 864745 between the Type 2 diabetes (T2DM) and Non-diabetic subject (NDM)

\begin{tabular}{|c|c|c|c|c|c|c|c|}
\hline Model & Genotype & NDM & T2DM & Odds ratio $(95 \% \mathrm{CI})$ & $P$ value & AIC & $\mathrm{BIC}$ \\
\hline \multirow[t]{3}{*}{ Codominant } & $\mathrm{T} / \mathrm{T}$ & $744(58.4 \%)$ & $736(61.6 \%)$ & 1.00 & 0.140 & 3423.1 & 3452.1 \\
\hline & $\mathrm{C} / \mathrm{T}$ & $471(37 \%)$ & $397(33.2 \%)$ & $0.85(0.72-1.01)$ & & & \\
\hline & $\mathrm{C} / \mathrm{C}$ & $59(4.6 \%)$ & $61(5.1 \%)$ & $1.05(0.72-1.52)$ & & & \\
\hline \multirow[t]{2}{*}{ Dominant } & $\mathrm{T} / \mathrm{T}$ & $744(58.4 \%)$ & $736(61.6 \%)$ & 1.00 & 0.096 & 3422.3 & 3445.5 \\
\hline & $\mathrm{C} / \mathrm{T}-\mathrm{C} / \mathrm{C}$ & $530(41.6 \%)$ & $458(38.4 \%)$ & $0.87(0.74-1.02)$ & & & \\
\hline \multirow[t]{2}{*}{ Recessive } & $\mathrm{T} / \mathrm{T}-\mathrm{C} / \mathrm{T}$ & $1215(95.4 \%)$ & $1133(94.9 \%)$ & 1.00 & 0.560 & 3424.7 & 3447.9 \\
\hline & $\mathrm{C} / \mathrm{C}$ & $59(4.6 \%)$ & $61(5.1 \%)$ & $1.11(0.77-1.61)$ & & & \\
\hline \multirow[t]{2}{*}{ Overdominant } & $\mathrm{T} / \mathrm{T}-\mathrm{C} / \mathrm{C}$ & $803(63 \%)$ & $797(66.8 \%)$ & 1.00 & 0.049 & 3421.1 & 3444.4 \\
\hline & $\mathrm{C} / \mathrm{T}$ & $471(37 \%)$ & $397(33.2 \%)$ & $0.85(0.72-1.00)$ & & & \\
\hline Log-additive & --- & -- & -- & $0.92(0.81-1.06)$ & 0.240 & 3423.6 & 3446.9 \\
\hline
\end{tabular}

Note: Significant threshold after Bonferroni correction for multiple comparisons is less than 0.010 (0.05/5); AIC: Akaike information criterion; BIC: Bayesian information criterion; CI: confidence interval.

Table 7. Different inheritance models analysis of the SNP rs35767 between the Type 2 diabetes (T2DM) and Non-diabetic subject (NDM)

\begin{tabular}{|c|c|c|c|c|c|c|c|}
\hline Model & Genotype & NDM & T2DM & Odds ratio $(95 \% \mathrm{CI})$ & $P$ value & AIC & $\mathrm{BIC}$ \\
\hline \multirow[t]{3}{*}{ Codominant } & $\mathrm{G} / \mathrm{G}$ & $500(39.2 \%)$ & $513(43 \%)$ & 1.00 & 0.110 & 3422.7 & 3451.7 \\
\hline & $\mathrm{G} / \mathrm{A}$ & $597(46.9 \%)$ & $512(42.9 \%)$ & $0.84(0.70-0.99)$ & & & \\
\hline & $\mathrm{A} / \mathrm{A}$ & $177(13.9 \%)$ & $169(14.2 \%)$ & $0.93(0.73-1.19)$ & & & \\
\hline \multirow[t]{2}{*}{ Dominant } & $\mathrm{G} / \mathrm{G}$ & $500(39.2 \%)$ & $513(43 \%)$ & 1.00 & 0.061 & 3421.5 & 3444.8 \\
\hline & G/A-A/A & $774(60.8 \%)$ & $681(57 \%)$ & $0.86(0.73-1.01)$ & & & \\
\hline \multirow[t]{2}{*}{ Recessive } & G/G-G/A & $1097(86.1 \%)$ & $1025(85.8 \%)$ & 1.00 & 0.820 & 3425.0 & 3448.2 \\
\hline & $\mathrm{A} / \mathrm{A}$ & $177(13.9 \%)$ & $169(14.2 \%)$ & $1.03(0.82-1.29)$ & & & \\
\hline \multirow[t]{2}{*}{ Overdominant } & G/G-A/A & $677(53.1 \%)$ & $682(57.1 \%)$ & 1.00 & 0.044 & 3421.0 & 3444.2 \\
\hline & $\mathrm{G} / \mathrm{A}$ & $597(46.9 \%)$ & $512(42.9 \%)$ & $0.85(0.72-1.00)$ & & & \\
\hline Log-additive & --- & 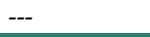 & 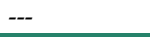 & $0.93(0.83-1.04)$ & 0.220 & 3423.5 & 3446.8 \\
\hline
\end{tabular}

Note: Significant threshold after Bonferroni correction for multiple comparisons is less than 0.010 (0.05/5); AIC: Akaike information criterion; BIC: Bayesian information criterion; CI: confidence interval.

Table 8. The haplotype analysis for SNPs located in intron 2 of the IGF2BP2 gene between the Type 2 diabetes (T2DM) and Non-diabetic subject (NDM)

\begin{tabular}{|c|c|c|c|c|c|}
\hline Haplotypes (rs4376068-rs4402960-rs6769511) & T2DM (frequency) & NDM (frequency) & $\mathrm{Chi}^{2}$ & $P$ value & Odds ratio $(95 \% \mathrm{CI})$ \\
\hline CTC & $592.48(0.248)$ & $562.60(0.221)$ & 5.923 & 0.015 & $1.179[1.033 \sim 1.346]$ \\
\hline AGT & $1690.21(0.708)$ & $1892.36(0.743)$ & 5.923 & 0.015 & $0.848[0.743 \sim 0.969]$ \\
\hline
\end{tabular}

Note: Significant threshold after Bonferroni correction for multiple comparisons is less than 0.025 (0.05/2); CI: confidence interval.

\section{Discussion}

We investigated the influence of 5 SNPs in 3 genes (rs864745 in JAZF1, rs35767 in IGF1, and rs4376068, rs4402960, and rs6769511 in IGF2BP2) on
T2DM in a Chinese population. These SNPs contribute to insulin resistance by affecting lipid metabolism in T2DM.

IGF2BP2 has a comprehensive role in lipid metabolism $[13,14]$. Dai et al. reported that IGF2BP2 
null mice are hypolipidemic, glucose tolerant, and insulin sensitive [13]. The loss of IGF2BP2 results in an increase in uncoupling protein-1 (UCP1), which contributes to resistance to obesity and a beneficial metabolic profile [13]. Long et al. reported the association of the rs6769511C allele in IGF2BP2 with the risk of T2DM in African Americans [22]. Kuo et al. also observed that the rs6769511C allele was associated with T2DM in a Chinese Han population [26]. Haljas et al. identified an association between the rs6769511C allele and T2DM in a meta-analysis [37]. Our current results also indicate an association between the allele and genotypes of rs6769511 with T2DM. The $C$ allele of rs6769511 was implicated as a risk factor for the development of T2DM ( $\mathrm{P}=0.009$, $\mathrm{OR}=1.185 ; 95 \%$ CI: 1.043-1.347). Horikawa et al. and Omori et al. reported the association of rs4402960T with the risk of developing T2DM in Japanese populations [20, 21]. Wu et al. and Huang et al. also found that the rs4402960T allele plays an important role in the predisposition of T2DM in the Chinese population [38, 39]. Several subsequent studies reported the association of the rs4402960T allele with the risk of T2DM in different populations [31, 40-42]. One of the reasons for the association of rs4402960T with T2DM could be the association of the T allele of rs4402960 with insulin sensitivity, which affects all glucose tolerance phenotypes, or the significantly increased insulin resistance of genotype TT, determined by homoeostasis model assessment for insulin resistance (HOMA-IR) and increased levels of IGF2BP2 mRNA in adipocytes [5, 6]. Therefore, rs4402960T could lead to disorders of T2DM related metabolic traits by increasing IGF2BP2 mRNA levels. In the current study, we also found that the $\mathrm{T}$ allele of rs4402960 could be a risk for T2DM (P=0.011, $\mathrm{OR}=1.181$; 95\% CI: 1.039-1.342). However, after Bonferroni correction, the SNP showed no significant difference between T2DM and NDM. One of the reasons for the discrepancies between the current and prior results could be the sample size. Our sample size was relatively moderate. We calculated the statistical power of rs4402960 using PS software [36] and it reached 0.487. If our sample size was greater, such as 2000 cases and 2000 controls, the statistical power would reach 0.746 . Thus, future studies should include more subjects to conclusively identify the association between these SNPs and T2DM. Moreover, in the current study, we observed that the $C$ allele of rs4376068 could be a risk for T2DM $(\mathrm{P}=0.022)$. There was no significant difference between T2DM and NDM after Bonferroni correction. However, since no other studies have addressed the association between rs4376068 and T2DM, our results must be confirmed in other studies.
In the current study, the haplotypes rs4376068Crs4402960T-rs6769511C and rs4376068A-rs4402960Grs6769511T in IGF2BP2 acted as risk and protective haplotypes in T2DM, respectively. Interestingly, the haplotypes that included the risk or protective alleles of the three SNPs were also risk and protective haplotypes in T2DM. These findings indicate that the three SNPs in the polymorphic genes may have combinatorial effects on T2DM susceptibility. In addition, compared with the effect of a single locus, the role of haplotypes on complex T2DM may be more remarkable. The reason for the positive haplotype association with T2DM and not the single locus could be that the single locus has a weak effect on the disease, while the linkage of loci has a reinforcing effect. Christodoulou and Avgeris et al. recently investigated the expression patterns of T2DM susceptibility genes and determined their individual transcript variants (tv) in peripheral blood of T2DM patients and controls ( $\mathrm{CT}_{\mathrm{RF}-}$ group: no risk factors for the development of $\mathrm{T} 2 \mathrm{D} ; \mathrm{CT}_{\mathrm{RF}}$ group: risk factors for the disease). Their data showed that the levels of tv7 of IGF2BP2 (which lacks exons 1 and 2 compared to the canonical tv1) exhibited a significant stepwise upregulation from $\mathrm{CT}_{\mathrm{RF}-}$ to $\mathrm{CT}_{\mathrm{RF}+}$ in $\mathrm{T} 2 \mathrm{DM}$ patients. Since rs4376068, rs4402960, and rs6769511 were all located in intron 2 of IGF2BP2, these SNPs could influence the transcript variants of IGF2BP2, which was associated with the development of T2DM [43].

JAZF1 serves as a metabolic regulator with the function of improving lipid metabolism and resisting hyperglycaemia through multiple metabolic signalling pathways in T2DM [16]. The favourable effects of JAZF1 on lipid metabolism have been observed in adipocytes and hepatocytes $[15,17,18]$. In addition to its role in improving lipid metabolism, JAZF1 has an important role in promoting glucose transport and enhancing insulin sensitivity in both liver and adipose tissue [7]. Several studies have reported an association between the $\mathrm{T}$ allele and TT genotype of rs864745 with the development of T2DM in African American, ethnic Chinese She, Japanese, and Uyghur populations [12, 23, 24, 29, 44]. However, Deng et al. did not find an association between rs864745 and T2DM risk in a Chinese Han population [24], which is consistent with the current results. Similarly, Gamboa-Meléndez et al. found no association between rs864745 and T2DM in a Mexican Mestizo population ( $\mathrm{P}>0.05)$ [45]. One of the reasons for the study differences could be the differences in ethnic and genetic backgrounds.

IGF1 is a key factor supporting growth and regulating metabolism. Nearly 30 years ago, Schalch et al. reported that IGF-1 was associated with significant reductions in fasting blood glucose and 
serum insulin [19]. More recently, Dupuis et al. documented that the association of the rs35767G allele in IGF1 was associated with HOMA-IR and T2DM in a European population [10]. Dotta et al. reported that the rs35767GG genotype exhibited circulating concentrations of IGF1 and lower insulin sensitivity in Europeans [11]. However, Zhang et al. found that the A allele of rs35767 contributed to the risk of developing T2DM in a Chinese Han population [25]. Presently, we did not find evidence of an association between rs35767 and T2DM. One of the reasons for the discrepancies in the risk allele of rs35767 between European and Chinese populations could be the genetic background. The frequency of rs35767A was approximately $25 \%$ to $37 \%$ in the Asian population, but only $16 \%$ in the European population (https:// asia.ensembl.org/Homo_sapiens/Variation/Populati on?db=core; $r=12: 102481291-102482291 ; \mathrm{v}=\mathrm{rs} 35767 ; \mathrm{vdb}$ $=$ variation; $\mathrm{vf}=95676582)$. The discrepancy between our data and that of Zhang et al. could be due to the different sample sizes. A total of 1,194 cases and 1,274 controls were enrolled in the current study. Zhang et al. enrolled 244 cases and 142 controls. Genetic differences could be another reason for this. The samples in the current study were from Yunnan Province, southwest China. The sample in the study by Zhang et al. was from Hebei province in the north of China. Several studies have reported that the genetic background is distinct between northern and southern Han, as identified using short tandem repeats, SNP, and HLA genes [46-49]. Moreover, the risks for the same disease in the Han Chinese population involving certain SNPs could be different between the northern and southern Han.

One limitation of the current study is that a relatively moderate sample size may limit its statistical power; the statistical power for the effect of the rs6769511 SNP in IGF2BP2 was 0.512, which was relatively lower. Thus, a larger population should be investigated, and the results of the current study validated in future studies.

\section{Conclusions}

In the current study, we focused on SNPs of three genes (JAZF1, IGF1, and IGF2BP2) which have been shown to affect lipid metabolism and insulin resistance. Our results revealed a significant association between rs6769511 in IGF2BP2 with T2DM, indicating that the SNP could be related to insulin resistance involving lipid metabolism. Moreover, the rs4376068-rs4402960-rs6769511 haplotypes in IGF2BP2 was associated with the development of T2DM. The results indicated that the functional effects of these polymorphisms associated with lipid metabolism and insulin resistance need to be investigated further.

\section{Acknowledgments}

This work was supported by grants from the National Science Foundation of China (31660313 and 81760734), The Association Foundation Program of Yunnan Provincial Science and Technology Department and Kunming Medical University (2019FE001-092), Reserve Talents of Young and Middle-aged Academic Leaders in Yunnan Province (2018HB047), Special Funds for High-level Health Talents of Yunnan Province (2017040), Yunnan Provincial Prevention and Treatment of Diabetic Vascular Disease Innovation Team (2019HC002), and the Clinical Medical Center of Endocrinology and Metabolic Disease of Yunnan Province (ZX2019-0202).

\section{Competing Interests}

The authors have declared that no competing interest exists.

\section{References}

1. Cai X, Xia L, Pan Y, He D, Zhu H, Wei T, et al. Differential role of insulin resistance and beta-cell function in the development of prediabetes and diabetes in middle-aged and elderly Chinese population. Diabetology \& metabolic syndrome. 2019; 11: 24 .

2. DeFronzo RA, Tripathy D. Skeletal muscle insulin resistance is the primary defect in type 2 diabetes. Diabetes Care. 2009; 32 Suppl 2: S157-63.

3. Johnson AM, Olefsky JM. The origins and drivers of insulin resistance. Cell. 2013; 152: 673-84.

4. Perry RJ, Samuel VT, Petersen KF, Shulman GI. The role of hepatic lipids in hepatic insulin resistance and type 2 diabetes. Nature. 2014; 510: 84-91.

5. Ruchat S-M, Elks CE, Loos RJF, Vohl M-C, Weisnagel SJ, Rankinen T, et al. Association between insulin secretion, insulin sensitivity and type 2 diabetes susceptibility variants identified in genome-wide association studies. Acta Diabetologica. 2008; 46: 217-26.

6. Chistiakov DA, Nikitin AG, Smetanina SA, Bel'chikova LN, Suplotova LA, Shestakova MV, et al. The rs11705701 G>A Polymorphism of IGF2BP2 is Associated With IGF2BP2 mRNA and Protein Levels in the Visceral Adipose Tissue - A Link to Type 2 Diabetes Susceptibility. The Review of Diabetic Studies. 2012; 9: 112-22.

7. Yuan L, Luo X, Zeng M, Zhang Y, Yang M, Zhang L, et al. Transcription factor TIP27 regulates glucose homeostasis and insulin sensitivity in a PI3-kinase/Akt-dependent manner in mice. International journal of obesity. 2015; 39: 949-58.

8. Li X, Yang M, Wang H, Jia Y, Yan P, Boden G, et al. Overexpression of JAZF1 protected ApoE-deficient mice from atherosclerosis by inhibiting hepatic cholesterol synthesis via CREB-dependent mechanisms. International Journal of Cardiology. 2014; 177: 100-10.

9. Thankamony A, Capalbo D, Marcovecchio ML, Sleigh A, Jørgensen SW, Hill NR, et al. Low circulating levels of IGF-1 in healthy adults are associated with reduced $\beta$-cell function, increased intramyocellular lipid, and enhanced fat utilization during fasting. The Journal of clinical endocrinology and metabolism. 2014; 99: 2198-207.

10. Dupuis J, Langenberg C, Prokopenko I, Saxena R, Soranzo N, Jackson AU, et al. New genetic loci implicated in fasting glucose homeostasis and their impact on type 2 diabetes risk. Nature genetics. 2010; 42: 105-16.

11. Dotta F, Mannino GC, Greco A, De Lorenzo C, Andreozzi F, Marini MA, et al. A Fasting Insulin-Raising Allele at IGF1 Locus Is Associated with Circulating Levels of IGF-1 and Insulin Sensitivity. PloS one. 2013; 8: e85483.

12. Langberg KA, Ma L, Sharma NK, Hanis CL, Elbein SC, Hasstedt SJ, et al. Single nucleotide polymorphisms in JAZF1 and BCL11A gene are nominally associated with type 2 diabetes in African-American families from the GENNID study. Journal of Human Genetics. 2011; 57: 57-61.

13. Dai N, Zhao L, Wrighting D, Krämer D, Majithia A, Wang Y, et al. IGF2BP2/IMP2-Deficient mice resist obesity through enhanced translation of Ucp1 mRNA and Other mRNAs encoding mitochondrial proteins. Cell metabolism. 2015; 21: 609-21.

14. Regué L, Minichiello L Liver-specific deletion of IGF2 mRNA binding protein-2/IMP2 reduces hepatic fatty acid oxidation and increases hepatic triglyceride accumulation. J Biol Chem. 2019; 294: 11944-51. 
15. Ming GF, Xiao D, Gong WJ, Liu HX, Liu J, Zhou HH, et al. JAZF1 can regulate the expression of lipid metabolic genes and inhibit lipid accumulation in adipocytes. Biochemical and biophysical research communications. 2014; 445: 673-80.

16. Liao ZZ, Wang YD, Qi XY, Xiao XH. JAZF1, a relevant metabolic regulator in type 2 diabetes. Diabetes Metabolism Research and Reviews. 2019; 35.

17. Wei Q, Zhou B, Yang G. JAZF1 ameliorates age and diet-associated hepatic steatosis through SREBP-1c -dependent mechanism. 2018; 9: 859.

18. Li L, Yang Y, Yang G, Lu C, Yang M, Liu H, et al. The role of JAZF1 on lipid metabolism and related genes in vitro. Metabolism. 2011; 60: 523-30.

19. Schalch DS, Turman NJ, Marcsisin VS, Heffernan M, Guler HP. Short-term effects of recombinant human insulin-like growth factor I on metabolic control of patients with type II diabetes mellitus. The Journal of clinical endocrinology and metabolism. 1993; 77: 1563-8.

20. Horikawa Y, Miyake K, Yasuda K, Enya M, Hirota Y, Yamagata K, et al. Replication of genome-wide association studies of type 2 diabetes susceptibility in Japan. The Journal of clinical endocrinology and metabolism. 2008; 93: 3136-41.

21. Omori S, Tanaka Y, Takahashi A, Hirose H, Kashiwagi A, Kaku K, et al. Association of CDKAL1, IGF2BP2, CDKN2A/B, HHEX, SLC30A8, and KCNJ11 with susceptibility to type 2 diabetes in a Japanese population. Diabetes. 2008; 57: 791-5.

22. Long J, Edwards T, Signorello LB, Cai Q, Zheng W, Shu XO, et al. Evaluation of genome-wide association study-identified type 2 diabetes loci in African Americans. American journal of epidemiology. 2012; 176: 995-1001.

23. Chen G, Xu Y, Lin Y, Lai X, Yao J, Huang B, et al. Association study of genetic variants of 17 diabetes-related genes/loci and cardiovascular risk and diabetic nephropathy in the Chinese She population. J Diabetes. 2013; 5: 136-45.

24. Deng X, Liu H, et al. Association of polymorphisms rs290487, rs864745, rs4430796 and rs23136 with type 2 diabetes in the Uyghur population in China. International journal of clinical and experimental pathology. 2017; 10: 8813-9.

25. Zhang J, Chen X, Zhang L, Peng Y. IGF1 gene polymorphisms associated with diabetic retinopathy risk in Chinese Han population. Oncotarget. 2017; 8: 88034-42.

26. Kuo JZ, Sheu WH, Assimes TL, Hung YJ, Absher D, Chiu YF, et al. Trans-ethnic fine mapping identifies a novel independent locus at the $3^{\prime}$ end of CDKAL1 and novel variants of several susceptibility loci for type 2 diabetes in a Han Chinese population. Diabetologia. 2013; 56: 2619-28.

27. American Diabetes A. 2. Classification and Diagnosis of Diabetes: Standards of Medical Care in Diabetes-2020. Diabetes Care. 2020; 43: S14-S31.

28. Alharbi KK, Ali Khan I, Syed R, Alharbi FK, Mohammed AK, Vinodson B, et al. Association of JAZF1 and TSPAN8/LGR5 variants in relation to type 2 diabetes mellitus in a Saudi population. Diabetology \& metabolic syndrome. 2015; 7: 92.

29. Ueyama M, Nishida N, Korenaga M, Korenaga K, Kumagai E, Yanai H, et al. The impact of PNPLA3 and JAZF1 on hepatocellular carcinoma in non-viral hepatitis patients with type 2 diabetes mellitus. J Gastroenterol. 2016; 51:370-9.

30. Wang R, Xu D, Liu R, Zhao L, Hu L, Wu P. Microsatellite and Single Nucleotide Polymorphisms in the Insulin-Like Growth Factor 1 Promoter with Insulin Sensitivity and Insulin Secretion. Med Sci Monit. 2017; 23: 3722-36.

31. Benrahma H, Charoute H, Lasram K, Boulouiz R, Atig RK, Fakiri M, et al. Association analysis of IGF2BP2, KCNJ11, and CDKAL1 polymorphisms with type 2 diabetes mellitus in a Moroccan population: a case-control study and meta-analysis. Biochemical genetics. 2014; 52: 430-42.

32. Li Y, Li C, Yang M, Shi L, Tao W, Shen K, et al. Association of single nucleotide polymorphisms of miRNAs involved in the GLUT4 pathway in T2DM in a Chinese population. Mol Genet Genomic Med. 2019; 7: e907.

33. Shi YY, He L. SHEsis, a powerful software platform for analyses of linkage disequilibrium, haplotype construction, and genetic association at polymorphism loci. Cell Res. 2005; 15: 97-8.

34. Li Z, Zhang Z, He Z, Tang W, Li T, Zeng Z, et al. A partition-ligationcombination-subdivision EM algorithm for haplotype inference with multiallelic markers: update of the SHEsis (http://analysis.bio-x.cn). Cell Res. 2009; 19: 519-23.

35. Sole X, Guino E, Valls J, Iniesta R, Moreno V. SNPStats: a web tool for the analysis of association studies. Bioinformatics. 2006; 22: 1928-9.

36. Dupont WD, Plummer WD, Jr. Power and sample size calculations for studies involving linear regression. Control Clin Trials. 1998; 19: 589-601.

37. Haljas K, Amare AT, Alizadeh BZ, Hsu YH, Mosley T, Newman A, et al. Bivariate Genome-Wide Association Study of Depressive Symptoms With Type 2 Diabetes and Quantitative Glycemic Traits. Psychosomatic medicine. 2018; 80: 242-51.

38. Wu Y, Li H, Loos RJ, Yu Z, Ye X, Chen L, et al. Common variants in CDKAL1, CDKN2A/B, IGF2BP2, SLC30A8, and HHEX/IDE genes are associated with type 2 diabetes and impaired fasting glucose in a Chinese Han population. Diabetes. 2008; 57: 2834-42

39. Huang Q, Yin J-y, Dai X-p, Pei Q, Dong M, Zhou Z-g, et al. IGF2BP2 variations influence repaglinide response and risk of type 2 diabetes in Chinese population. Acta Pharmacologica Sinica. 2010; 31: 709-17.

40. Gu T, Horova E, Mollsten A, Seman NA, Falhammar H, Prazny M, et al. IGF2BP2 and IGF2 genetic effects in diabetes and diabetic nephropathy. J Diabetes Complications. 2012; 26: 393-8.

41. Lasram K, Ben Halim N, Benrahma H, Mediene-Benchekor S, Arfa I, Hsouna S, et al. Contribution of CDKAL1 rs7756992 and IGF2BP2 rs4402960 polymorphisms in type 2 diabetes, diabetic complications, obesity risk and hypertension in the Tunisian population. J Diabetes. 2015; 7: 102-13.

42. Totomoch-Serra A, Munoz ML, Burgueno J, Revilla-Monsalve MC, Perez-Munoz A, Diaz-Badillo A. The ADRA2A rs553668 variant is associated with type 2 diabetes and five variants were associated at nominal significance levels in a population-based case-control study from Mexico City. Gene. 2018; 669: 28-34.

43. Christodoulou MI, Avgeris M. Blood-based analysis of type-2 diabetes mellitus susceptibility genes identifies specific transcript variants with deregulated expression and association with disease risk. Scientific Reports. 2019; 9: 1512.

44. Song M, Zhao F, Ran L, Dolikun M, Wu L, Ge S, et al. The Uyghur population and genetic susceptibility to type 2 diabetes: potential role for variants in CDKAL1, JAZF1, and IGF1 genes. Omics : a journal of integrative biology. 2015; 19: 230-7.

45. Gamboa-Meléndez MA, Huerta-Chagoya A, Moreno-Macías H, VázquezCárdenas P, Ordóñez-Sánchez ML, Rodríguez-Guillén R, et al. Contribution of common genetic variation to the risk of type 2 diabetes in the Mexican Mestizo population. Diabetes. 2012; 61: 3314-21.

46. Chu JY, Huang W, Kuang SQ, Wang JM, Xu JJ, Chu ZT, et al. Genetic relationship of populations in China. Proc Natl Acad Sci U S A. 1998; 95: 11763-8.

47. Su B, Xiao J, Underhill P, Deka R, Zhang W, Akey J, et al. Y-Chromosome evidence for a northward migration of modern humans into Eastern Asia during the last Ice Age. Am J Hum Genet. 1999; 65: 1718-24.

48. Shi L, Xu SB, Ohashi J, Sun H, Yu JK, Huang XQ, et al. HLA-A, HLA-B, and HLA-DRB1 alleles and haplotypes in Naxi and Han populations in southwestern China (Yunnan province). Tissue Antigens. 2006; 67: 38-44.

49. Yao Y, Shi L, Shi L, Matsushita M, Yu L, Lin K, et al. Distribution of HLA-A, -B, $-\mathrm{CW}$, and -DRB1 alleles and haplotypes in an isolated Han population in Southwest China. Tissue Antigens. 2009; 73: 561-8. 